\title{
Caracterización genética del porcino Criollo de Ecuador
}

\author{
Estupiñan, K. '; Barba, C. ${ }^{2}$; Martínez, A. ${ }^{3 \oplus}$ y Delgado, J.V. ${ }^{3}$
}

'Facultad de Ciencias Pecuarias. Universidad Técnica Estatal de Quevedo. Quevedo, Los Ríos. Ecuador. 2Departamento de Producción Animal. Universidad de Córdoba. Córdoba. España.

${ }^{3}$ Departamento de Genética. Universidad de Córdoba. Córdoba. España.

\section{PaLABRAS CLAVE}

Microsatélites.

Diversidad genética.

Iberoamérica.

Heterocigosis.

\section{RESUMEN}

Ecuador es un país con una elevada biodiversidad de recursos genéticos animales de interés agroalimentario, aunque la información cientifica sobre ellos es escasa. En este trabajo se aborda el estudio de la diversidad genética de los cerdos Criollos de la Zona de Planificación Cinco de Ecuador, conformada por las provincias de Bolívar, Guayas, Los Ríos y Santa Elena, en donde este cerdo juega un papel fundamental en las comunidades campesinas como la principal fuente de proteína de la unidad familiar y, como una fuente de ingresos complementarios en sistemas de producción tradicionales. Se analizan 90 muestras con 25 microsatélites recomendados por la FAO para estudios de diversidad genética porcina y se calculan los parámetros básicos de variabilidad genética (número medio de alelos, número efectivo de alelos, heterocigosis, PIC y coeficiente FIS). Se calculan las distancias genéticas DSA y se construye un dendrograma de distancias entre individuos. Los cerdos Criollos de Ecuador estudiados presentan una elevada diversidad genética intra-racial, no se desvían significativamente del Equilibrio de Hardy-Weinberg. No se detecta diferenciación genética entre los cerdos muestreados en las diferentes provincias por lo que se podría gestionar como una sola población.

\section{Genetic characterization of the Ecuadorian Creole pig}

\section{SUMMARY}

\section{ADDITIONAL KEYWORDS}

Microsatellites.

Genetic diversity.

Iberoamerica.

Heterocigosity.

INFORMATION

\section{Cronología del artículo.}

Recibido/Received: 04.05.2020

Aceptado/Accepted: 02.07.2020

On-line: 15.10 .2020

Correspondencia a los autores/Contact e-mail:

ib2mamaa@uco.es.

\section{INTRODUCCIÓN}

El patrimonio ganadero criollo constituye la base de la sustentabilidad de los sistemas tradicionales de producción animal en Iberoamérica, ya sea por la resiliencia y capacidad de adaptación de estas poblaciones animales a los hábitats naturales donde históricamente se han explotado, como por ofrecer un nivel de produc-
Ecuador is a country with a high biodiversity of animal genetic resources of agri-food interest, although scientific information on them is scarce. This work aims is the study of the genetic diversity of Creole pigs in the Planning Zone Five of Ecuador, integrated by the provinces of Bolivar, Guayas, Los Ríos and Santa Elena. There, this population plays a fundamental role in rural communities as the main source of protein for the family unit and as a source of complementary income in traditional production systems. Ninety diversity. Parameters of genetic variability are calculated (mean number of alleles, effective number of alleles, heterozygosity, PIC and FIS coefficient). Individual DSA genetic distances are calculated and a dendrogram is constructed. The studied Ecuadorian pig shows a high in-breed genetic diversity and it do not deviate significantly from the Hardy-Weinberg equilibrium. No genetic differentiation is detected between the pigs sampled in the different provinces, so it could be managed as a single population. samples are analyzed with 25 microsatellites recommended by the FAO for studies of porcine genetic ción aceptable con la implementación de tecnologías de bajo coste, así como los productos obtenidos, carne y grasa, fundamentalmente, forman parte de la cultura alimentaria de las comunidades rurales, contribuyendo de esta manera a alcanzar la soberanía alimentaria (Delgado et al. 2004). En este contexto, el ganado porcino criollo se erige como paradigma zootécnico en este ámbito, dadas algunas de sus ventajas competitivas: 
comportamiento alimentario omnívoro, que le permite el aprovechamiento de recursos naturales mediante pastoreo, así como de subproductos agroindustriales; elevada prolificidad, ciclo de vida corto, resistencia a enfermedades y diversidad de producciones (Barba et al. 2014).

Ecuador es un país muy biodiverso, tanto a nivel global como desde el punto de vista de los recursos genéticos animales de interés agroalimentario, si bien no abundan los estudios de caracterización genética de sus poblaciones criollas (FAO 2015), en especial la de la especie porcina, donde solo se registra un estudio preliminar sobre 15 ejemplares (Vargas et al. 2015), a diferencia de lo que ocurre en otros países de su entorno como Brasil (Sollero et al. 2009); Colombia (Gélvez et al. 2015), y Venezuela (Galíndez et al. 2016).

En la Zona de Planificación Cinco de Ecuador, conformada por las provincias de Bolívar, Guayas, Los Ríos y Santa Elena, el cerdo criollo se ha mantenido presente en las comunidades campesinas como la principal fuente de proteína de la unidad familiar y, eventualmente, como alternativa de ingresos complementarios en sistemas de producción de pequeña escala y de traspatio en un contexto de economía de subsistencia.

La información genética sobre las poblaciones porcinas criollas resulta esencial para conocer su situación desde el punto de vista de la conservación con vistas a instaurar programas de desarrollo ganadero (Delgado, 2012). Por esta razón, el análisis de la variabilidad genética permite valorar esta población como recurso genético y así poder determinar las estrategias a seguir para desarrollar un programa de cría compatible con el desarrollo sustentable. El objetivo principal de este estudio fue la caracterización genética del ganado porcino de la Zona de Planificación 5 de Ecuador con el fin de optimizar el diseño de un programa de desarrollo ganadero.

\section{MATERIAL Y MÉTODOS}

Se analizaron 90 muestras de pelo de cerdos Criollos de Ecuador recogidas en las provincias de Los Ríos, Guayas, Santa Elena y Bolívar (Figura 1). El ADN se extrajo utilizando el método de Walsh (Walsh, Metzger, y Higuchi 1991). Se amplificaron mediante PCR 25 microsatélites recomendados por la FAO para estudios de biodiversidad porcina (FAO 2011): CGA, IGF1, S0002, S0005, S0026, S0068, S0090, S0101, S0155, S0178, S0215, S0225, S0226, S0227, S0228, S0355, S0386, SW24, SW240, SW632, SW72, SW857, SW911, SW936 y SW951. Los fragmentos amplificados se separaron mediante electroforesis en gel de poliacrilamida en un secuenciador automático ABI 3130XL utilizando Genescan ${ }^{\circledR}$ 400HD ROX Size Standard (ThermoFisher Scientific, Massachusetts, USA). El análisis de fragmentos y la tipificación alélica se realizaron con el software Genescan Analysis $₫ 3.1 .2$ y Genotyper $₫$ 2.5.2, respectivamente (Applied Biosystems, Massachusetts, USA).

Se calculó el número medio de alelos por locus (MNA), las frecuencias alélicas, las heterocigosis esperada $(\mathrm{He})$ y observada $(\mathrm{Ho})$ y el contenido de informa-

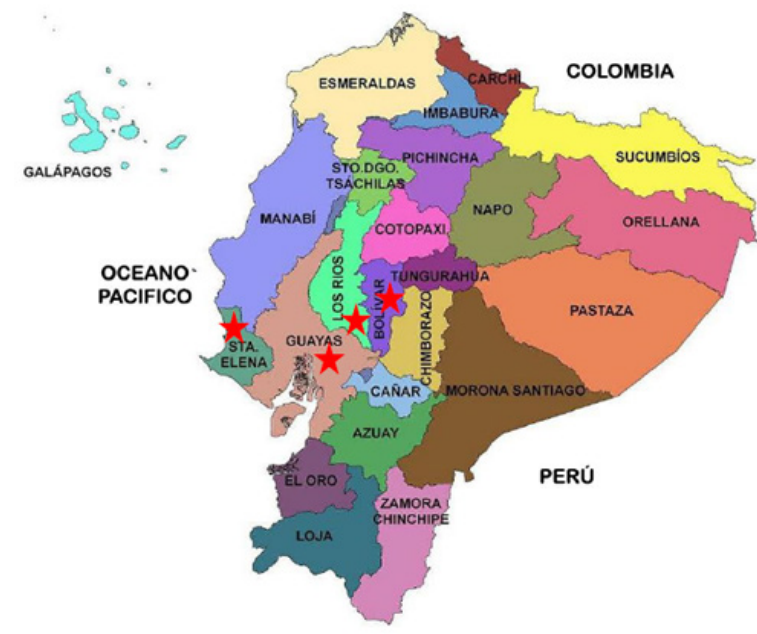

Figura 1. Provincias de Ecuador de procedencia de las muestras analizadas (Provinces of Ecuador from the provenance of the samples analysed).

ción polimórfica (PIC) con el programa MICROSATELLITE TOOLKIT software para Excel (Park 2001). Se obtuvo el número efectivo de alelos con el programa PopGene (Yeh et al. 1999). Los valores de $F_{\text {IS }}$ (coeficiente de consanguinidad) con un intervalo de confianza del $95 \%$ se calcularon con el programa informático GENETIX v. 4.05 (Belkhir et al. 2004) y se realizó una prueba de equilibrio Hardy-Weinberg (HWE) usando el programa GENEPOP v. 3.1c (Raymond \& Rousset, 1995), que aplica el test exacto de Fisher usando el método en cadena de Monte Carlo Markov (Guo \& Thompson 1992) y la corrección de Bonferroni. Para el cálculo de las distancias genéticas entre individuos DSA (Bowcock et al. 1994) se utilizó el programa Populations v. 1.2.28 (Langella 1999). El dendrograma se visualizó utilizando el programa TREEVIEW (Page R.D. 1996).

\section{RESULTADOS Y DISCUSIÓN}

Sin duda, el primer paso a dar en la conservación de las poblaciones animales es el conocimiento intrínseco de su estructura genética, además de sus relaciones genéticas con otras poblaciones potencialmente relacionadas (Alderson 2018). Este proceso de análisis intrapoblacional e interpoblacional se conoce como caracterización genética.

Diversos tipos de marcadores genéticos se han utilizado en todas las especies domésticas en este proceso, pero los microsatélites se han impuesto como los más utilizados, por su eficiencia y simplicidad. Su uso en la caracterización de razas locales de porcino ha tenido una gran relevancia en programas de conservación actuales (Jiménez et al. 2017; Margeta et al. 2018).

Nuestros resultados han mostrado a la población porcina estudiada como una entidad definida frente a otros grupos de la especie, con buena estabilidad genética y sin estructura interna.

En la Tabla I se recogen los valores obtenidos de Heterocigosis esperada, Heterocigosis observada, 
Contenido de Información Polimórfica (PIC), los valores del estadístico $\mathrm{F}_{\mathrm{IS}}$ con sus desviaciones estándar y los marcadores desviados del equilibrio HardyWeinberg. A partir de los valores de PIC obtenidos, se puede afirmar que la mayoría de los marcadores son muy informativos (PIC > 0,50), aunque los microsatélites S0215, S0227, S0355 y SW951 son medianamente informativos (PIC entre 0,25 y 0,50). Tras la corrección de Bonferroni, ningún marcador está desequilibrado significativamente del Equilibrio de Hardy-Weinberg en esta población. De los 25 microsatélites analizados, 6 detectan un exceso significativo de homocigotos (CGA, S0068, S0090, S0215, S0225 y S0355).

El promedio de alelos en una población (Tabla I) indica en cierta manera la variabilidad genética de las poblaciones. Este número medio de alelos es elevado $(10,96)$, aunque el número efectivo de alelos $(4,75)$ es sensiblemente inferior debido a que muchos de los alelos se encuentran con una frecuencia inferior al 1\%. Tanto el número medio de alelos como el número efectivo de alelos están por encima de la media mostrada por otras razas porcinas criollas de su mismo tronco originario (Revidatti et al. 2014) y de las razas porcinas europeas estudiadas por San Cristobal el al. (2006). En la Tabla I se recogen los valores de heterocigosis media esperada $(\mathrm{He}=0,729)$ y heterocigosis media por recuento directo $(\mathrm{Ho}=0,665)$ en esta población. El promedio de alelos y los valores de heterocigosis indican que los cerdos Criollos de Ecuador muestran una diversidad genética alta, con valores de diversidad genética similares a los encontrados por Fang et al. (2005) en algunas razas chinas. El valor de $\mathrm{F}_{\mathrm{IS}}$ con un intervalo de confianza al 95\% con 1000 remuestreos es de 0,089 $(0,055-0,111)$ y no es significativo, lo que indica que la población no muestra una desviación significativa del equilibrio Hardy-Weinberg.

Los cerdos Criollos de Ecuador presentan una elevada diversidad genética intra-racial, con valores de diversidad genética superiores a los de otras razas porcinas criollas (Revidatti et al. 2014). La raza no se desvía significativamente del Equilibrio de HardyWeinberg lo que en principio podría ser un dato favorable pues no se apreciaría ni exceso de homocigotos ni de heterocigotos.

Tabla I. Microsatélites analizados, número de alelos detectados, Número efectivo de alelos (Ae), Heterocigosidades esperada insesgada (He) y observada (Ho), Contenido de Información Polimórfica (PIC), valores de Fis, su intervalo de confianza y las desviaciones del equilibrio Hardy-Weinberg (HWEd) (Microsatellites analyzed, number of alleles detected, Effective number of alleles (Ae), Heterozygosities expected insame (He) and observed (Ho), Polymorphic Information Content (PIC), Fis values, their confidence interval and Hardy-Weinberg balance deviations (HWEdEd)).

\begin{tabular}{|c|c|c|c|c|c|c|c|c|}
\hline Microsatélite & $\mathrm{N}^{\circ}$ Alelos & $\mathrm{Ae}$ & $\mathrm{He}$ & Ho & PIC & $F_{1 S}$ & $F_{\text {IS }}$ IC & HWEd \\
\hline CGA & 28 & 13,26 & 0,930 & 0,835 & 0,920 & 0,102 & $(0,014-0,186)$ & NS \\
\hline IGF1 & 10 & 4,21 & 0,767 & 0,697 & 0,730 & 0,092 & $(-0,029-0,208)$ & NS \\
\hline S0002 & 12 & 5,97 & 0,837 & 0,759 & 0,813 & 0,095 & $(-0,016-0,199)$ & NS \\
\hline S0005 & 22 & 11,83 & 0,921 & 0,854 & 0,910 & 0,073 & $(-0,010-0,150)$ & NS \\
\hline S0026 & 7 & 3,24 & 0,695 & 0,644 & 0,645 & 0,073 & $(-0,066-0,206)$ & NS \\
\hline S0068 & 17 & 7,12 & 0,865 & 0,738 & 0,845 & 0,147 & $(0,038-0,249)$ & NS \\
\hline S0090 & 8 & 4,33 & 0,774 & 0,659 & 0,736 & 0,149 & $(0,022-0,274)$ & NS \\
\hline S0101 & 11 & 3,20 & 0,691 & 0,708 & 0,661 & $-0,025$ & $(-0,137-0,083)$ & NS \\
\hline S0155 & 8 & 4,43 & 0,779 & 0,722 & 0,745 & 0,073 & $(-0,040-0,180)$ & NS \\
\hline S0178 & 10 & 4,81 & 0,796 & 0,711 & 0,767 & 0,108 & $(-0,012-0,219)$ & NS \\
\hline S0215 & 6 & 1,84 & 0,459 & 0,357 & 0,400 & 0,224 & $(0,017-0,410)$ & NS \\
\hline S0225 & 12 & 3,24 & 0,695 & 0,578 & 0,668 & 0,170 & $(0,025-0,305)$ & NS \\
\hline S0226 & 13 & 4,75 & 0,794 & 0,744 & 0,764 & 0,062 & $(-0,052-0,173)$ & NS \\
\hline S0227 & 8 & 1,51 & 0,339 & 0,281 & 0,325 & 0,172 & $(-0,014-0,352)$ & NS \\
\hline S0228 & 10 & 3,15 & 0,686 & 0,644 & 0,657 & 0,061 & $(-0,071-0,189)$ & NS \\
\hline S0355 & 9 & 1,97 & 0,495 & 0,416 & 0,470 & 0,161 & $(0,006-0,306)$ & NS \\
\hline S0386 & 10 & 4,39 & 0,777 & 0,729 & 0,748 & 0,061 & $(-0,052-0,172)$ & NS \\
\hline SW24 & 10 & 4,93 & 0,802 & 0,736 & 0,780 & 0,083 & $(-0,037-0,194)$ & NS \\
\hline SW240 & 10 & 5,31 & 0,816 & 0,809 & 0,789 & 0,009 & $(-0,093-0,105)$ & NS \\
\hline SW632 & 13 & 5,87 & 0,834 & 0,756 & 0,810 & 0,095 & $(-0,014-0,200)$ & NS \\
\hline SW72 & 6 & 3,39 & 0,709 & 0,622 & 0,652 & 0,123 & $(-0,010-0,248)$ & NS \\
\hline SW857 & 8 & 4,44 & 0,779 & 0,739 & 0,745 & 0,053 & $(-0,059-0,164)$ & NS \\
\hline SW911 & 9 & 4,49 & 0,781 & 0,733 & 0,750 & 0,062 & $(-0,056-0,171)$ & NS \\
\hline SW936 & 11 & 5,59 & 0,826 & 0,782 & 0,798 & 0,054 & $(-0,054-0,155)$ & NS \\
\hline SW951 & 6 & 1,60 & 0,378 & 0,367 & 0,354 & 0,031 & $(-0,112-0,186)$ & NS \\
\hline Number & 10,96 & 4,75 & 0,729 & 0,665 & 0,699 & & & \\
\hline
\end{tabular}




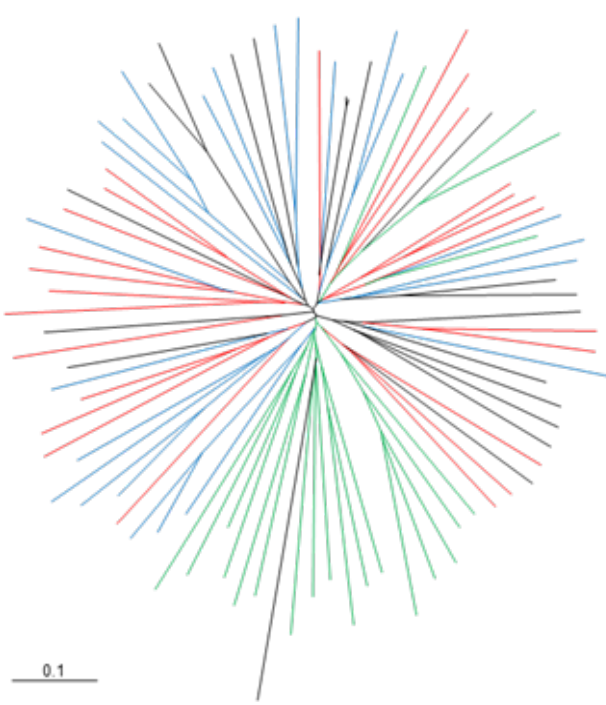

LOS RIOS

GUAYAS

SANTA ELENA

BOLIVAR

Figura 2. Árbol de distancias individuales DSA de los individuos de cerdos Criollos de Ecuador muestreados en cuatro provincias de Ecuador (DSA individual distance tree of Ecuador's Creole pig individuals sampled in four provinces of Ecuador).

En la Figura 2 está representado el dendrograma de distancias individuales entre los individuos analizados. No se observa un agrupamiento claro de los individuos en función de la provincia de procedencia, aunque la mayoría de los individuos de Santa Elena se agrupan (en verde en la figura 2 ).

A la vista de estos resultados podemos afirmar que los cerdos criollos muestreados en las provincias de Los Ríos, Guayas, Santa Elena y Bolívar de Ecuador constituyen una entidad genética bien definida y estable, pudiendo recomendarse su reconocimiento como raza, por sí misma, o integrada en una entidad nacional de distribución más amplia.

Teniendo en cuenta que una de las situaciones de más grande amenaza para una población es el desconocimiento de la misma, recomendamos que se implemente sobre esta población un programa de gestión genética adecuado a las necesidades técnicas demandadas.

\section{CONCLUSIÓN}

Los cerdos Criollos de Ecuador presentan una elevada diversidad genética intra-racial, no se desvían significativamente del Equilibrio de Hardy-Weinberg lo que en principio podría ser un dato favorable para abordar un plan eficiente de recuperación y conservación. No se detecta diferenciación genética entre los cerdos muestreados en las diferentes provincias por lo que se podría gestionar como una sola población.

\section{AGRADECIMIENTOS}

Los autores quieren agradecer el apoyo recibido por la empresa de base tecnológica de la Universidad de Córdoba Animal Breeding Consulting S.L. con el procesamiento de las muestras.

\section{BIBLIOGRAFÍA}

Alderson, GLH 2018, 'Conservación de razas y mantenimiento de la biodiversidad: justificación y metodología para la conservación de los recursos genéticos animales', Archivos de Zootecnia, vol. 67, no. 258, pp. 300-309.

Barba, C, León, JM, Gámiz, P, Delgado \& JV 2014, 'Historia de los cerdos en Iberoamérica y el Caribe', in Silva, FOL., (ed.), Las razas porcinas iberoamericanas: un enfoque etnozootécnico. IF. Baiano Salvador, BA. Brasil. pp. 23-36.

Belkhir, K, Borsa, P, Chikhi, L, Raufaste, N \& Bonhomme, F 2004, GENETIX 4.05, logiciel sous Windows TM pour la génétique des populations. Laboratoire Génome, Populations, Interactions, CNRS UMR 5000, Université de Montpellier II, Montpellier (France).

Bowcock, AM, Ruiz-Linares, A, Tomfohrde, J, Minch, E, Kidd, JR \& Cavalli-Sforza, LL 1994, 'High resolution of human evolutionary trees with polymorphic microsatellites', Nature, vol. 368, no. 6470, pp. 455-457.

Delgado, JV, Camacho, ME, León, JM, De la Haba, M, Vallecillo, A, Barba, C, \& Cabello, A 2004, 'Poblaciones porcinas iberoamericanas', in Delgado, JV (ed.), Biodiversidad Porcina lberoamericana. Caracterización y uso sustentable. Córdoba. España.

Delgado, J 2012, 'Conservación y utilización de los recursos genéticos de los animales de granja', Actas Iberoamericanas de Conservación Animal, vol. 2, $n^{\circ} 1$, pp. 19-23.

Fang, $M, H u, X$, Jiang, T, Braunschweig, $M, H u, L, D u, Z$, Feng, J, Zhang, Q, Wu, C \& Li, N 2005, 'The phylogeny of Chinese indigenous pig breeds inferred from microsatellite markers', Animal Genetics, vol. 36, no. 1, pp. 7-13.

FAO 2015, The second report on the state of the world's animal genetic resources for food and agriculture, FAO Commission on Genetic Resources for Food and Agriculture Assessments, Rome.

FAO 2011, Molecular genetic characterization of animal genetic resources, FAO Animal Production and Health Guidelines, Rome, <http://www.fao.org/docrep/014/i2413e/i2413e00.htm>.

Galíndez, R, Ramis, C, Martínez, G, Ángulo, L, Bedoya, Á \& de Farías, Y 2016, 'Variabilidad genética de seis poblaciones del cerdo Criollo venezolano usando marcadores microsatélites', Revista de la Facultad de Agronomía, vol. 42, no. 2, <http://190.169.94.12/ojs/ index.php/rev_agro/article/view/11901>, viewed 1 July 2020.

Gélvez, IM, Pérez, EP \& Martinez, TIC 2015, 'Variación genética en cerdo doméstico (Sus scrofa domestica) de Córdoba-Colombia basada en marcadores microsatélites', Revista Mexicana de Ciencias Pecuarias, vol. 6, no. 4, pp. 443-452.

Guo, SW \& Thompson, EA 1992, 'Performing the exact test of HardyWeinberg proportion for multiple alleles', Biometrics, vol. 48, no. 2, pp. 361-372.

Jiménez, ÁP, Albarracín, M \& Estupiñán, S 2017, 'Variabilidad genética del cerdo Congo Santandereano mediante marcadores microsatelite', Archivos de Zootecnia, vol. 66, no. 256, pp. 599-602.

Langella, O 1999, Populations 1.2.31: a population genetic software. CNRS UPR9034, <http://bioinformatics.org/ tryphon/populations/>, viewed 10 November 2012.

Margeta, P, Gvozdanovic, K, Kušec, ID, Radiši凶, Ž, Kusek, G \& Margeta, $\vee 2018$, 'Genetic analysis of Croatian autochthonous pig breeds based on microsatellite markers', Archivos de zootecnia, no. 1, pp. 13-16.

Page R.D. 1996, 'TreeView: an application to display phylogenetic trees on personal computers.', Computer Applications in the Biosciences, vol. 12, pp. 357-8.

Park, SDE 2001, 'Trypanotolerance in West African Cattle and the Population Genetic Effects of Selection', University of Dublin.

Revidatti, MA, Delgado Bermejo, JV, Gama, LT, Periati, VL, Ginja, C, Alvarez, LA, Vega-Pla, JL, Martínez, AM \& Consortium, B 2014, 'Genetic characterization of local Criollo pig breeds from the Americas using microsatellite markers', Journal of Animal Science, vol. 92, no. 11 , pp. 4823-4832. 
SanCristobal, M, Chevalet, C, Haley, CS, Joosten, R, Rattink, AP, Harlizius, B, Groenen, M a. M, Amigues, Y, Boscher, M-Y, Russell, G, Law, A, Davoli, R, Russo, V, Désautés, C, Alderson, L, Fimland, E, Bagga, M, Delgado, JV, Vega-Pla, JL, Martinez, AM, Ramos, M, Glodek, P, Meyer, JN, Gandini, GC, Matassino, D, Plastow, GS, Siggens, KW, Laval, G, Archibald, AL, Milan, D, Hammond, K \& Cardellino, R 2006, 'Genetic diversity within and between European pig breeds using microsatellite markers', Animal Genetics, vol. 37, no. 3, pp. 189-198.

Sollero, BP, Paiva, SR, Faria, DA, Guimarães, SEF, Castro, STR, Egito, AA, Albuquerque, MSM, Piovezan, U, Bertani, GR \& Mariante, A da S 2009, 'Genetic diversity of Brazilian pig breeds evidenced by microsatellite markers', Livestock Science, vol. 123, no. 1, pp. 8-15. Vargas, JC, Velázquez, FJ, Galíndez, R, Pérez-Pineda, E, PonceAlvarado, AP, Sierra-Vazquez, AC, Llambí, S, Montenegro, $M$, Álvarez, LA, Revidatti, MA; Matínez, MA, Landi, V, Delgado, JV, Carril, JA, \& Chacón, E 2015, 'Estructura y relaciones genéticas del cerdo criollo de Ecuador', Revista Electrónica Veterinaria, vol. 16, n 7 , pp. 1-12.

Yeh, FC, Yang, RC, Boyle, T, Ye, ZH \& Mao, JX 1999, 'POPGENE, version 1.32: the user-friendly software for population genetic analysis', <https://www.scienceopen.com/document?vid=c020f786-97ac-4fc4b81f-48b13b62d56a>, viewed 29 March 2019. 